\section{Effect of ECT on insulin}

SIR: we are pleased that Williams et al (Journal, July 1992, 161,94-98) have confirmed our finding (Berrios et al, 1986) that ECT causes a temporary elevation of plasma insulin, the more so for recent animal work (in which Dr Nutt also participated) seemed to show that this was not the case (Thiagarajan et al, 1988). However, contrary to what Dr Williams et al have reported, we found that 'the higher the ordinal position of ECT (in a given treatment course) the higher the peak of insulin at ten minutes' (p. 223). This discrepancy can only be resolved by further research.

At the time of our original work we had also: (a) compared subjects with and without anticholinergic premedication to ascertain whether vagal stimulation was involved, and ascertained that insulin was not elevated in subjects so premedicated, (b) found a similar elevation of insulin in patients with DSM-III major depression. (However, following the advice of the Editor that these findings might confuse the issue, we deleted them from the final version of the paper.)

The fact that a post-ECT elevation of insulin is found both in schizophrenia and in depression suggests that the response is unlikely to be specifically related to the neurobiology of depression.

Berrios, G. E., Bulbena, A., Martinez, A. R., et al (1986) Significant rise in plasma insulin after electroconvulsive therapy. Acta Psychiatrica Scandinavica, 74, 222-224.

Thiagarajan, A. B., Gletter, C. H. \& Nutt, D. J. (1988) Electroconvulsive shock does not increase plasma insulin in rats. Convulsive Therapy, 4, 292-296.

University of Cambridge

Department of Psychiatry

Addenbrooke's Hospital

Cambridge CB2 $2 Q Q$

\section{Reliability of DISCUS rating in individuals with learning disabilities}

SIR: Jones (Journal, September 1991, 159, 441-442) drew attention to the lack of reliability of the DISCUS rating (Sprague et al, 1989) in the assessment of motor disorders, particularly of tardive dyskinesia in individuals with learning disabilities. We, therefore, undertook to examine the inter-rater reliability of DISCUS ratings in 29 in-patients with learning disabilities. Each individual was independently assessed by two raters. Inter-rater reliability was assessed using the method of Brennan \& Silman (1992).

Pearson's product-moment correlation between scores was high $(t=+0.70, P<0.001)$. However, there was a difference in mean scores obtained by each rater of +3.24 (s.d. $2.68, t=2.05$ ). This results in a $95 \%$ range for agreement between observers of -2.25 to 8.73 . The 'true' value of the mean difference was considered as a measure of bias between observers and was obtained by calculating $95 \%$ confidence intervals. From the samples studied, the $95 \%$ confidence interval for the mean difference was found to be 1.36 to 5.12 . As zero lay outside this interval it was concluded that significant bias existed between observers.

Studies purporting to demonstrate a relationship between tardive dyskinesia, medication, and other indices in this group of individuals must take into account difficulties in objective assessment and significant bias between observers.

Brennan, P. \& Silman, A. (1992) Statistical methods of assessing observer variability in clinical measures. British Medical Journal, 304, 1491-1494.

Sprague, R. L., Kalachinik, J. E. \& Shaw, K. W. (1989) Psychometric properties of the dyskinesia identification system: condensed user scale (DISCUS). Mental Retardation, 27, 141-148.

\section{Leicester Frith Hospital \\ Groby Road \\ Leicester LE3 9QF}

\section{R. Collacott S. BHAUMIK D. BRANFORD}

\section{Evaluation in mental health care}

SIR: I am sure Freeman (Journal, July 1992, 161, 1-3) is not suggesting that evaluation is an impossibility in mental-health care. Everyone will accept that there are extensive difficulties in identifying appropriate methods for evaluation in psychiatry because of the nature of the problems presented by patients and the effect of the environment in which the services are provided. Surely, however, these problems are not unique to mental health as the same arguments could be put forward for the services to elderly people and children. What is needed, therefore, is an understanding of the complex problems associated with evaluation of mental-health services before pronouncements are made as it appears to be the case in Dr King's (1991) report.

Evaluation has become an essential part of the new National Health Service reforms and there is an increased expectation that the effectiveness and efficiency of health-care services is undertaken by professionals. Hence so much emphasis on medical audit, clinical audit, and research. Exciting opportunities are created as the result of the implementation of National Health Service/Community Care 